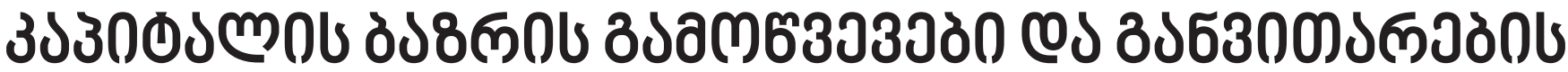

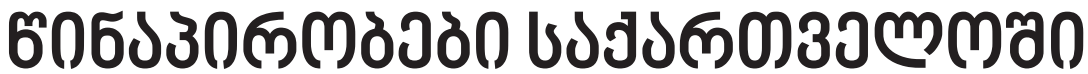

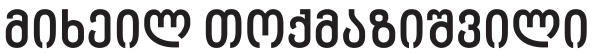

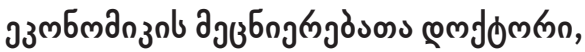

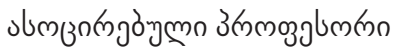

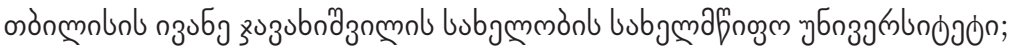

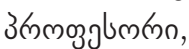

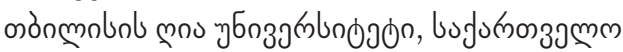

M-Tokmazishvili@yahoo.com

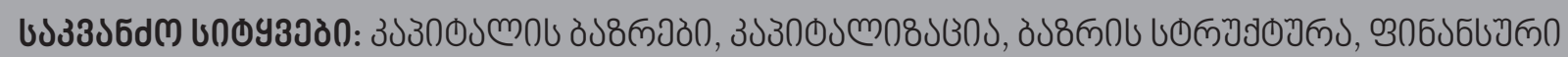

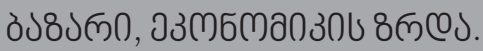

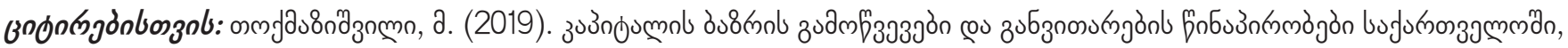

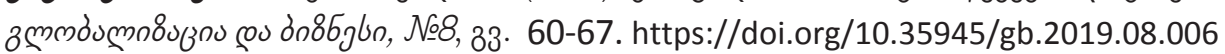

\section{ฆอเ১з১ल0}

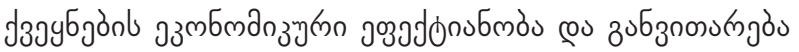

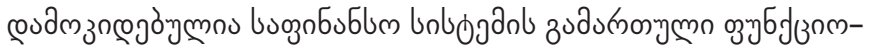

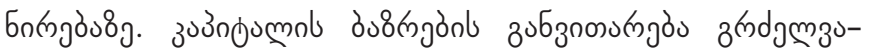

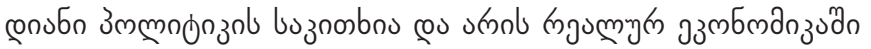

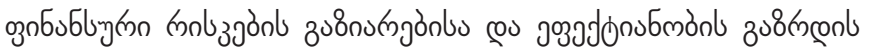

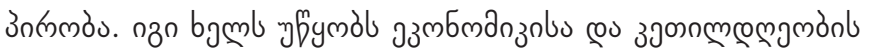
3u8moul.

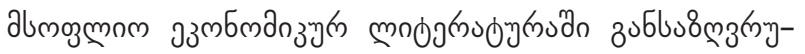

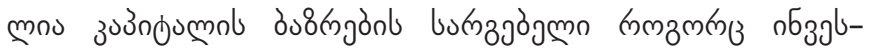

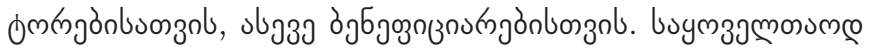

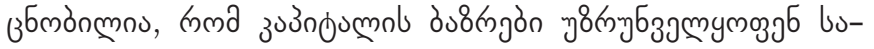

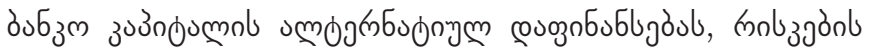

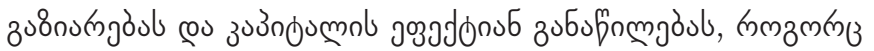

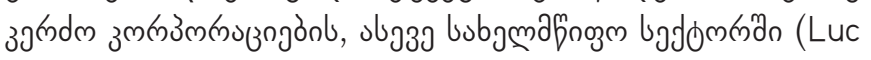
Laeven, 2014: 4-6).

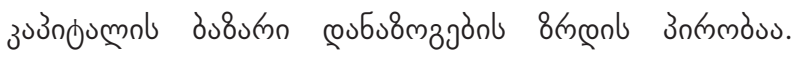

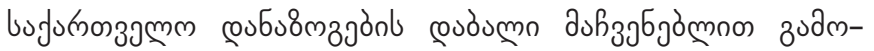

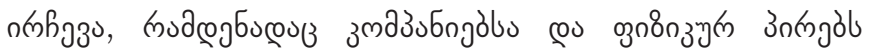

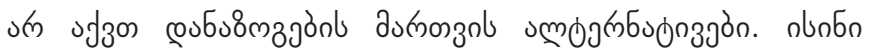

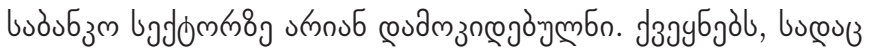

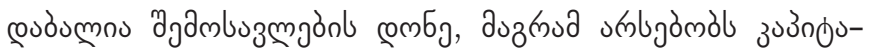

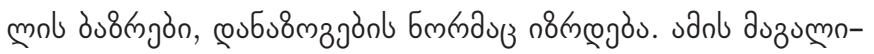

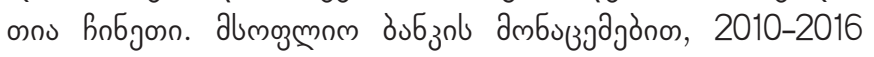

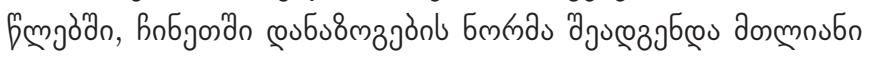

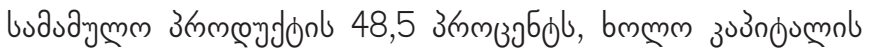

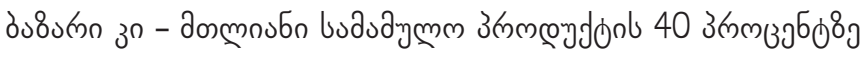

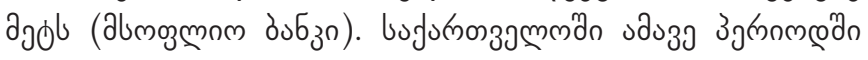

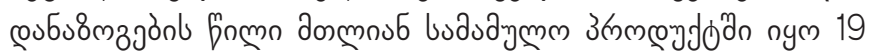

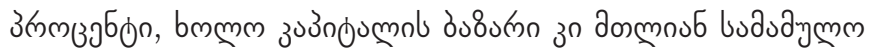

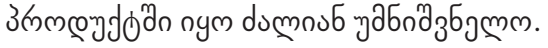

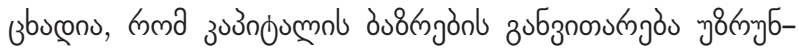

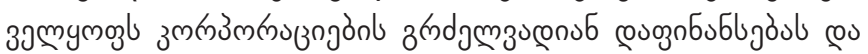

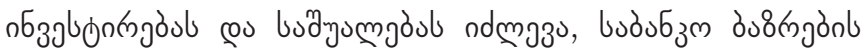

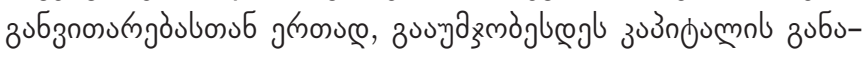

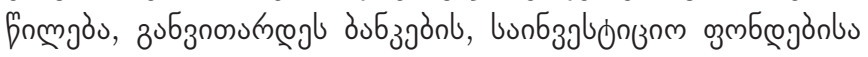

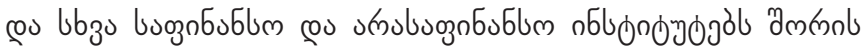

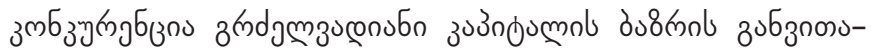

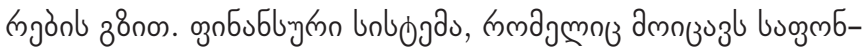

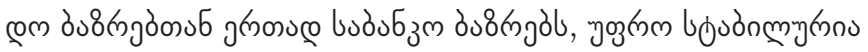

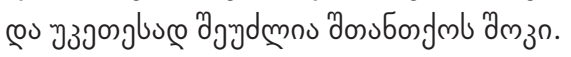

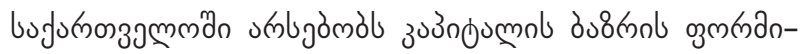

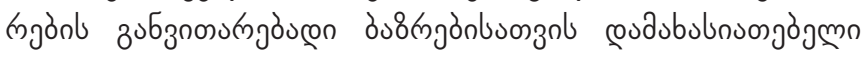

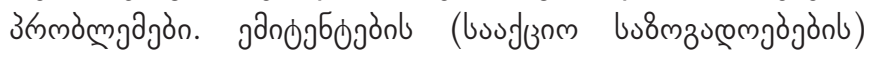

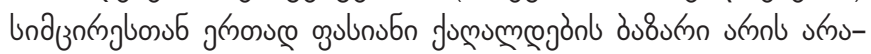

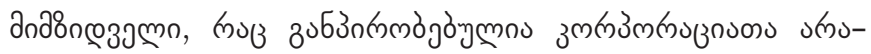

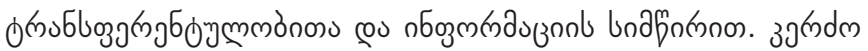

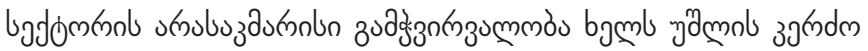
ugdom ñan nб

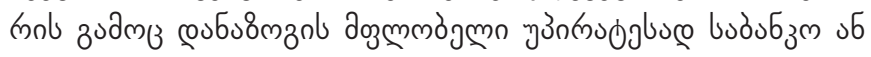

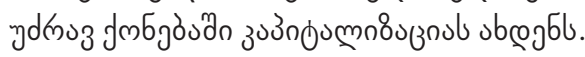

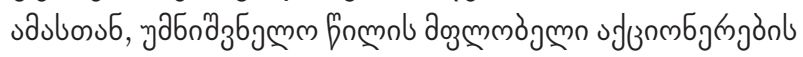

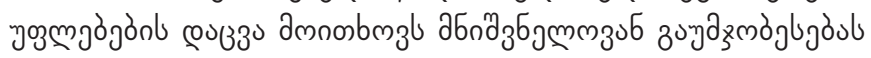




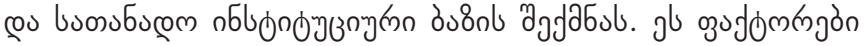

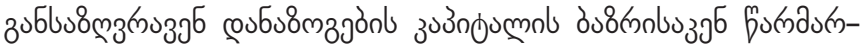

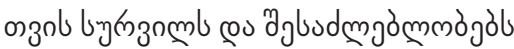

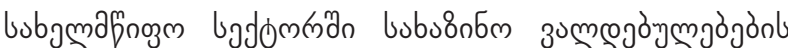

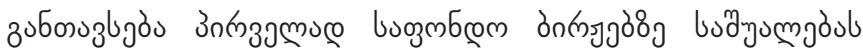

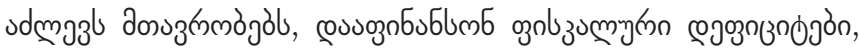

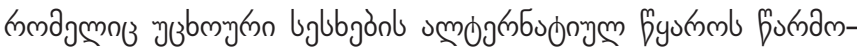
ง

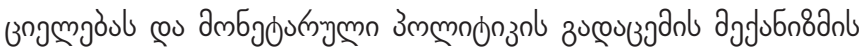
3uydzmògljobl (Monetary Policy Implementation at Different Stages of Market Development, 2004), Jabn b aзzmm-

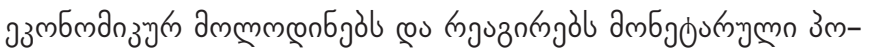

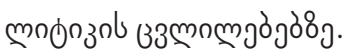

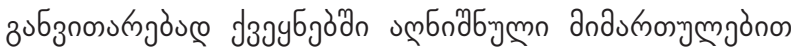

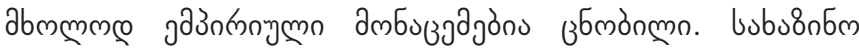

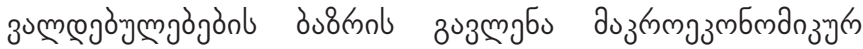
a

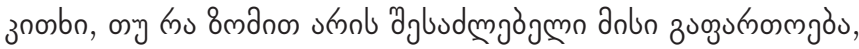

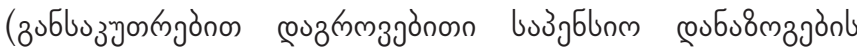

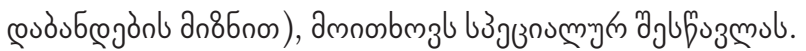

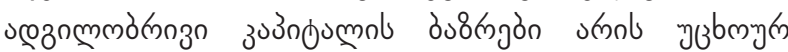

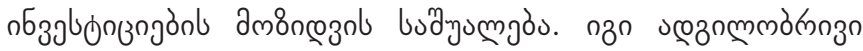

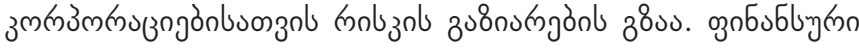

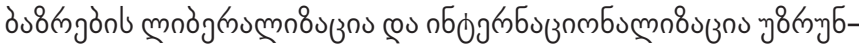

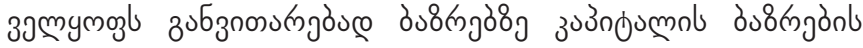

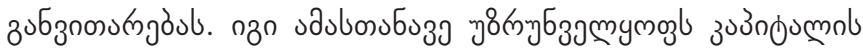

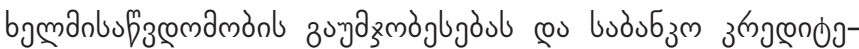

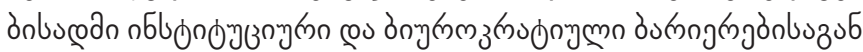

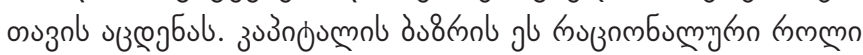

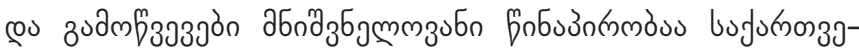

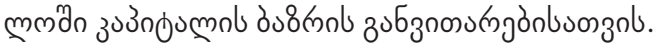

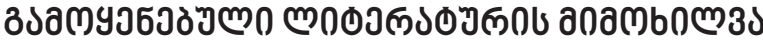

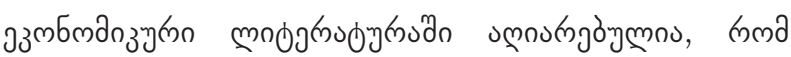

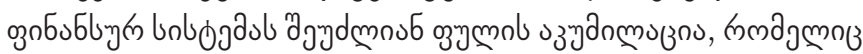

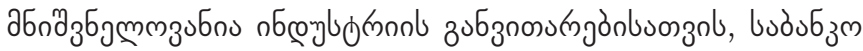

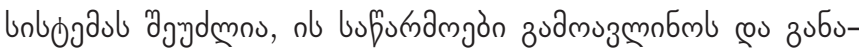

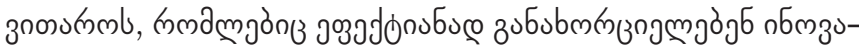

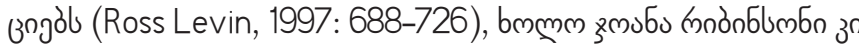

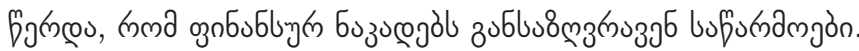

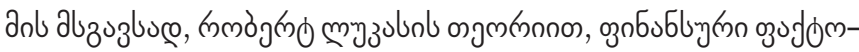

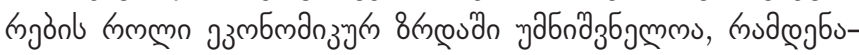

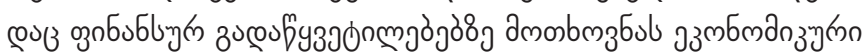

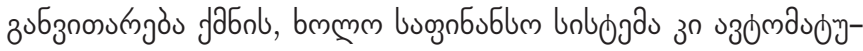

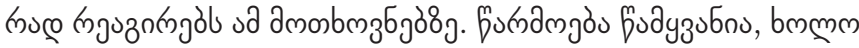

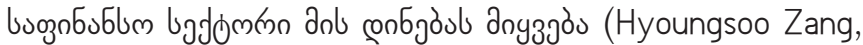
Young Chu Kim: 2007; Jagadish Prasad Bist, 2018).

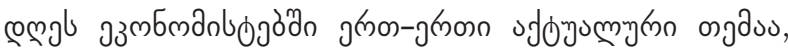

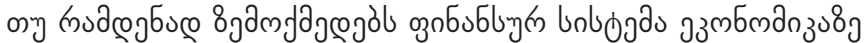
po kn zm

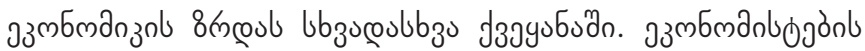

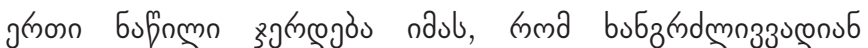

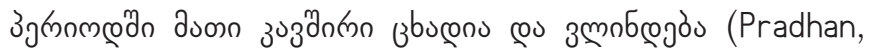
R.P., Arvin, M..B., Bahmani, S., Hall, J.H., Norman, N.,

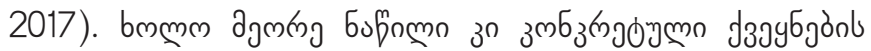

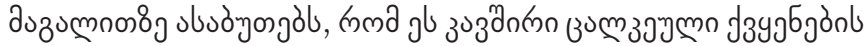

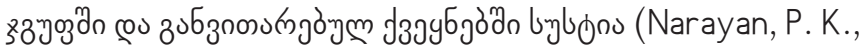
Narayan, S. 2013:70-78; Ayadi, R., Arbak, E., Naceur, S. B., De Groen, W. P, 2013; Ductor, L., Grechyna, D. , 2015: 393405; Grassa, R., Gazdar, K: 2014: 493-514; Mhadhbi, K. 2014 :

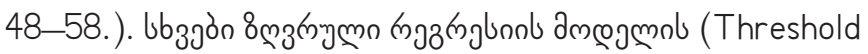

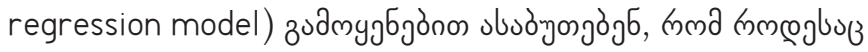

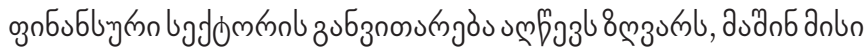

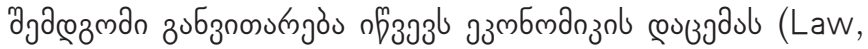
S. H., Singh, N., 2014: 36-44; Samargandi, N., Fidrmuc, J., Ghosh, S. 2015: 66-81.; Arcand, J. L., Berkes, E., Panizza, U. 2012; Rousseau, P. L., \& Wachtel, P. 2008; Demetriades,

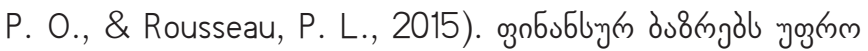

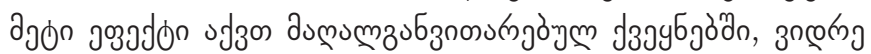

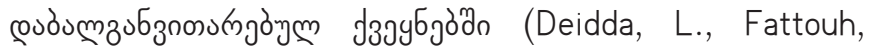

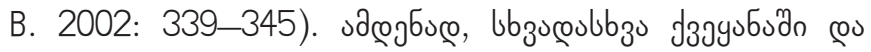
ذзэyб

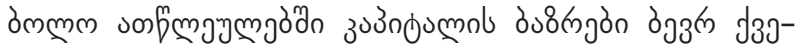

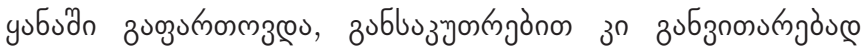

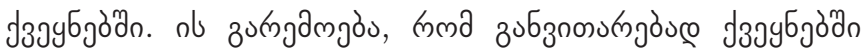

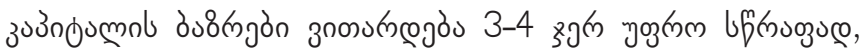

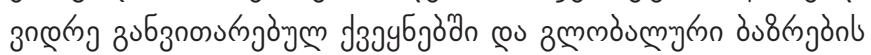

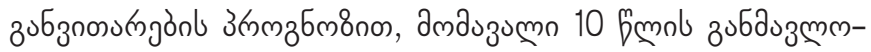

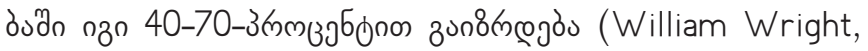
Panagiotis Asimakopoulos, Eivind Friis Hamre, 2019), dgo-

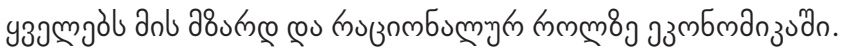

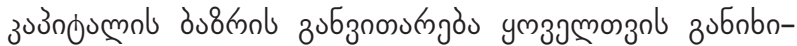

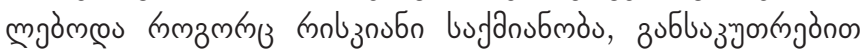

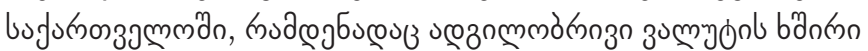

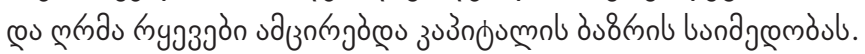

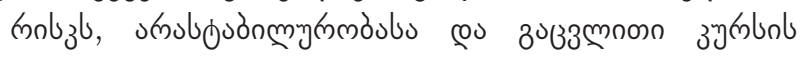

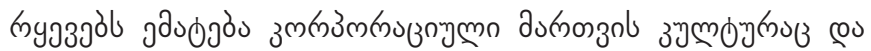

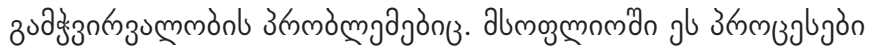

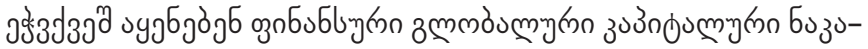

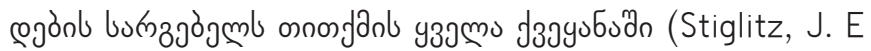
2002; Arcand, J. L., Berkes, E., Panizza, U, 2012). gl buznosbon

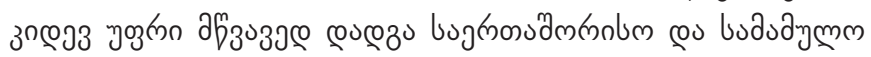

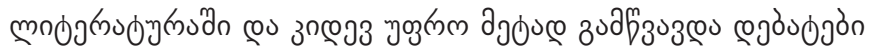

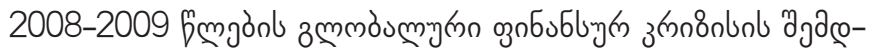

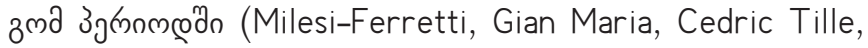
2011: 289-346; Lane, Philip R., Gian Maria Milesi-Ferretti,

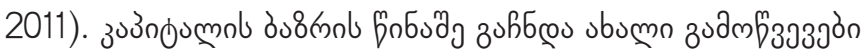

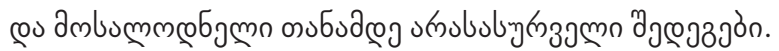




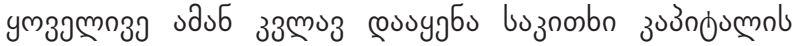

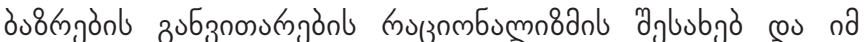

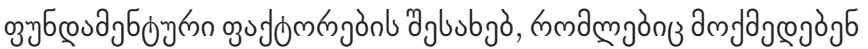

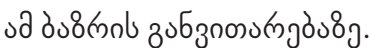

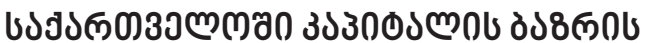

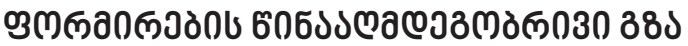

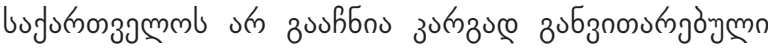

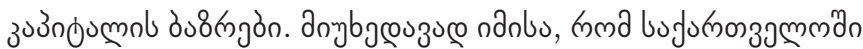

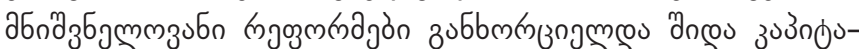

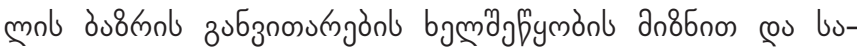

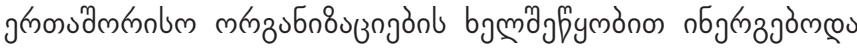

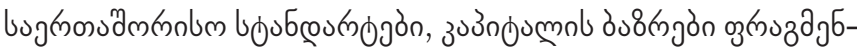

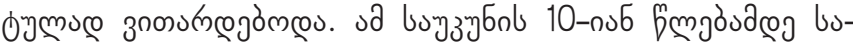

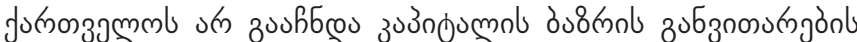

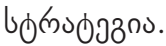

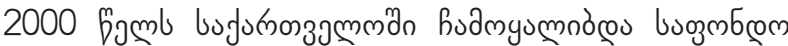

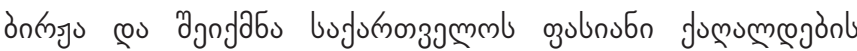

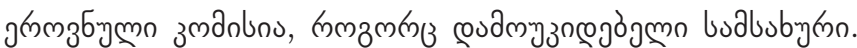

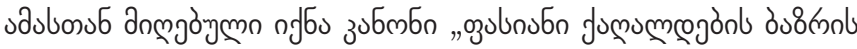

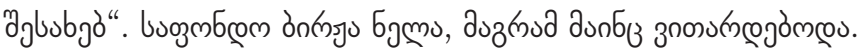

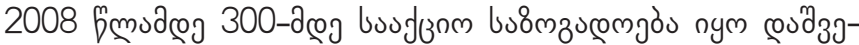

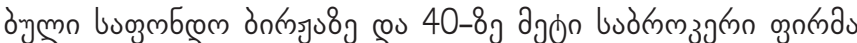

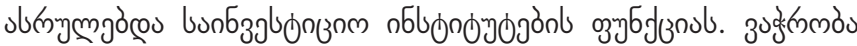

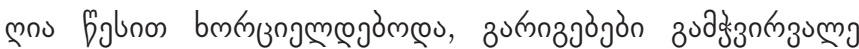

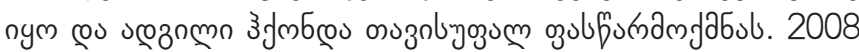

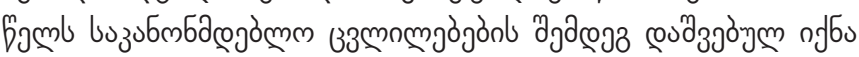

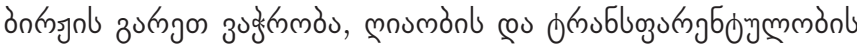

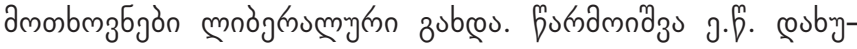

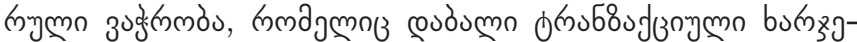

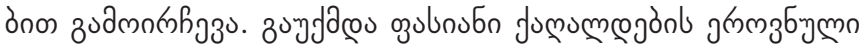

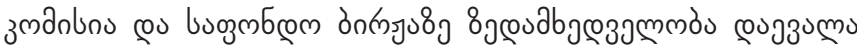

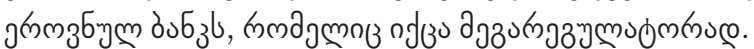

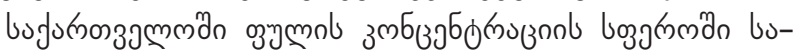

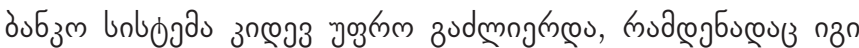

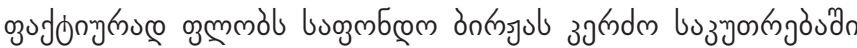

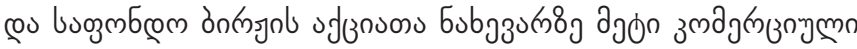

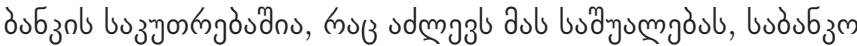
зuЗ

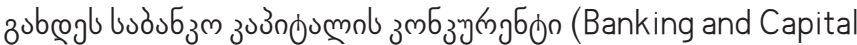
Markets M\&A Outlook, 2019)'.

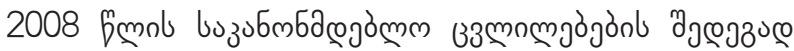

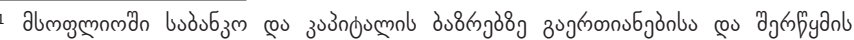

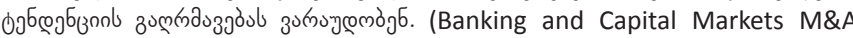
Outlook, Delloitte, Will it be a stellar year for banking and capital markets mergers and acquisitions? 2019

https://www2.deloitte.com/us/en/pages/financial-services/articles/banking-securities-mergers-acquisitions-outlook.html)
}

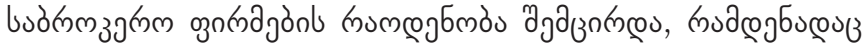

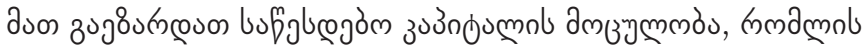

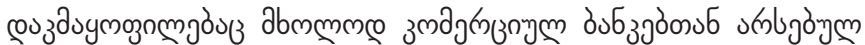

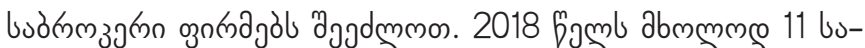

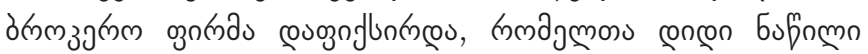

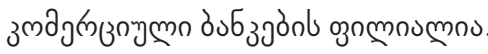

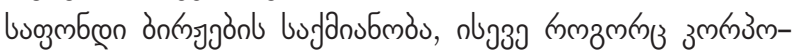

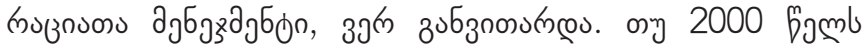

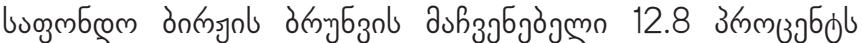

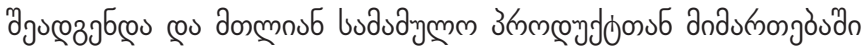

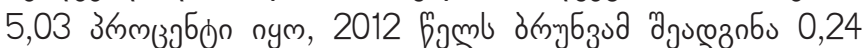

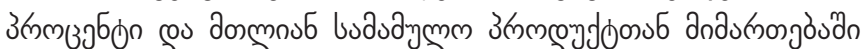

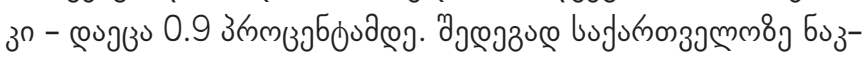

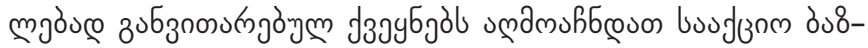

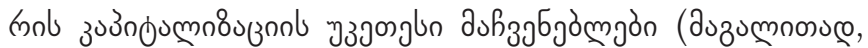

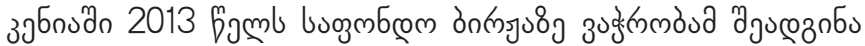

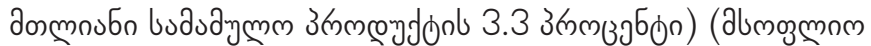
$\left.30 \sigma_{30}\right)$

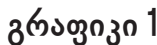

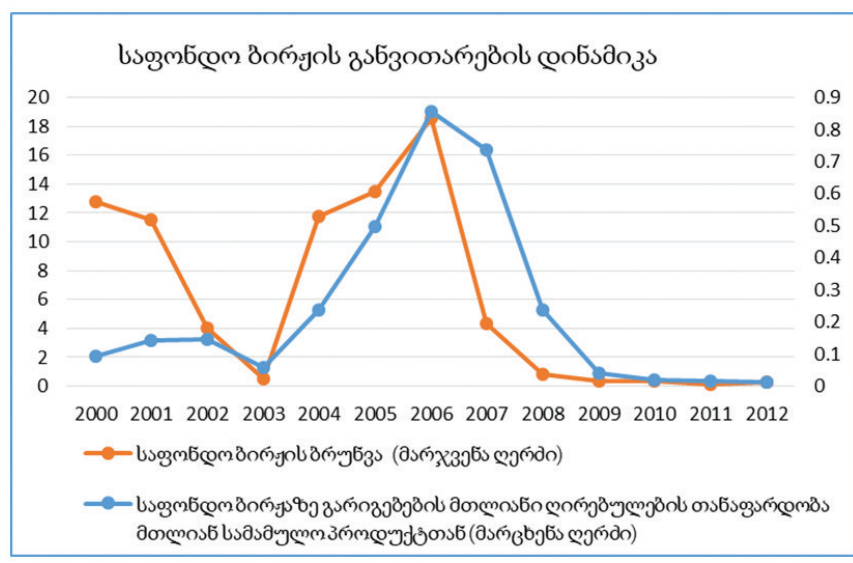

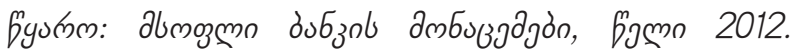
https://databank.worldbank.org/data/source/globalfinancial-development/Type/TABLE/preview/ onNoadvancedDownloadOptions

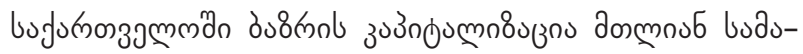

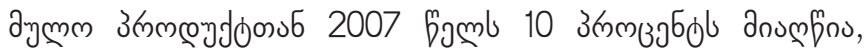

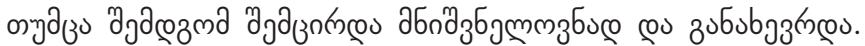

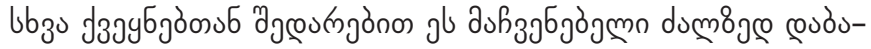

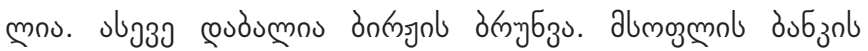

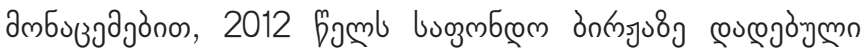

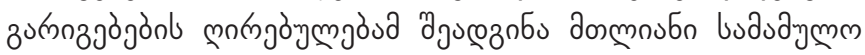

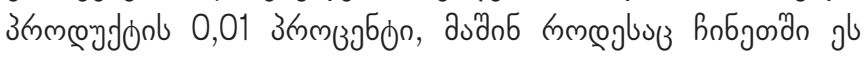

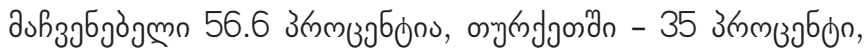

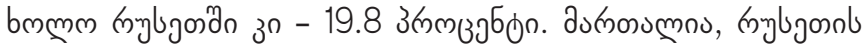

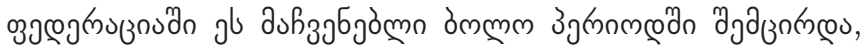

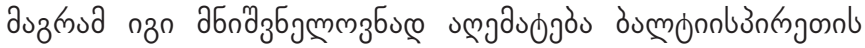

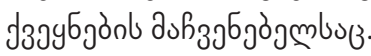




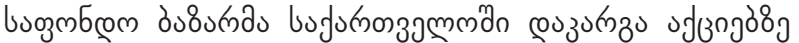

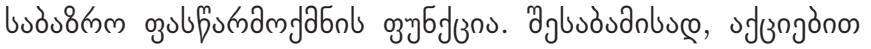

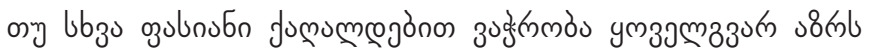

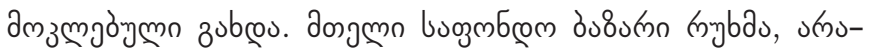

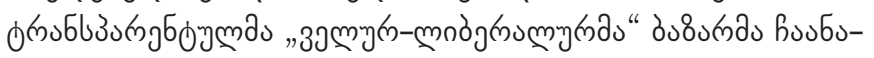
उзmo (Aslanishvili).

bufurnoszagmman nym zeos, bufurnoszamm zodbeounnym

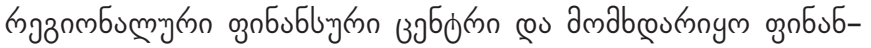

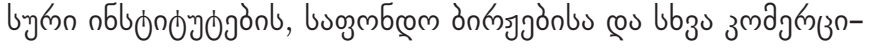

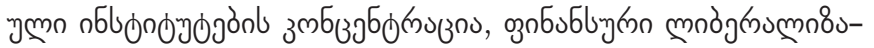

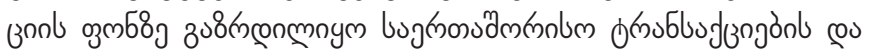

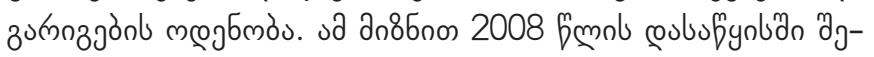

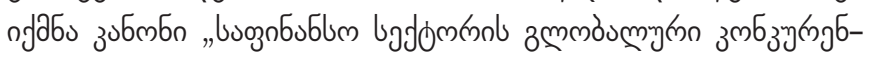

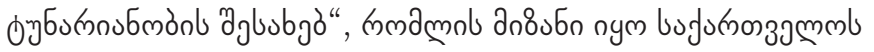

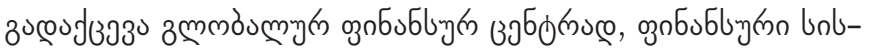
ojan

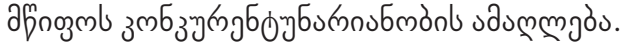

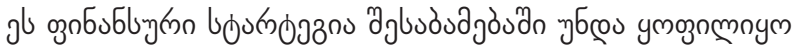

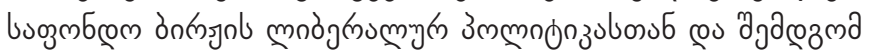

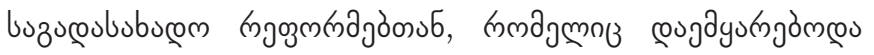

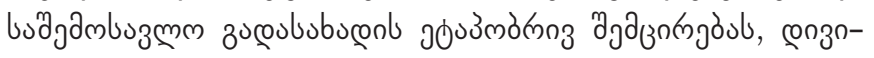

\section{3mogुnzo 2}

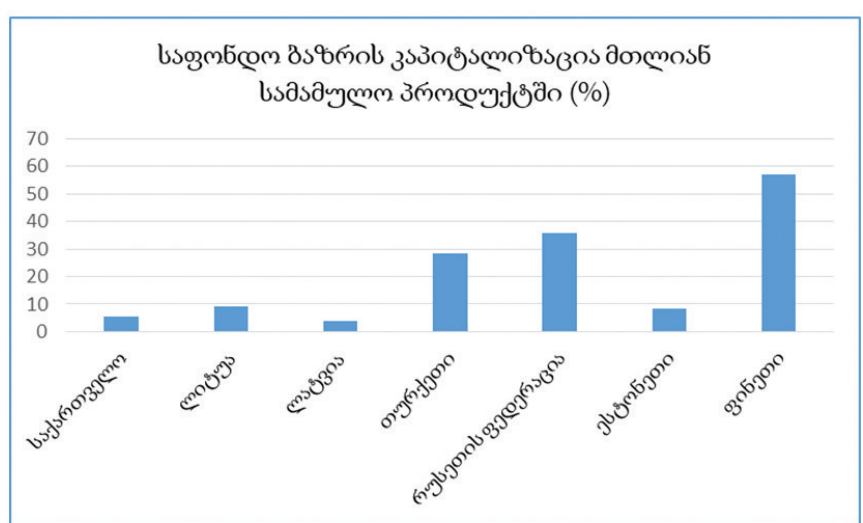

3mogुnzos

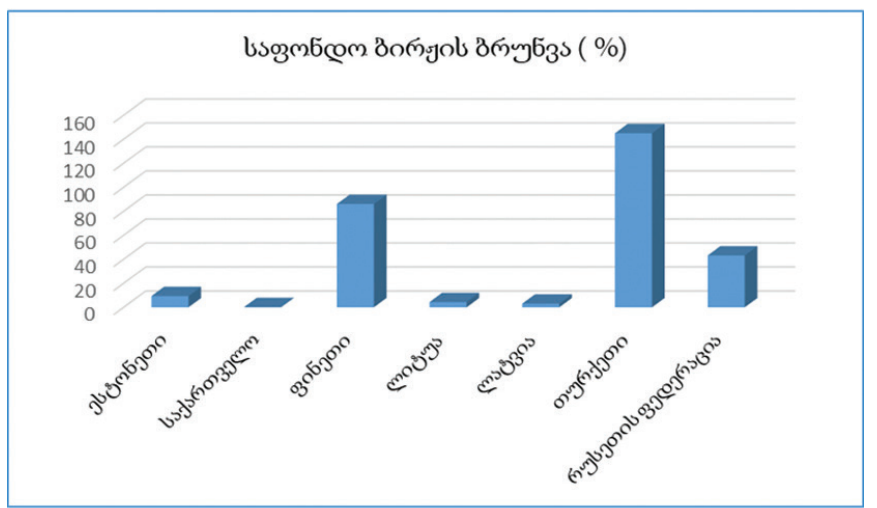

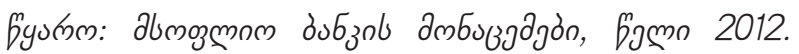
https://databank.worldbank.org/data/source/globalfinancial-development/Type/TABLE/preview/ onNoadvancedDownloadOptions

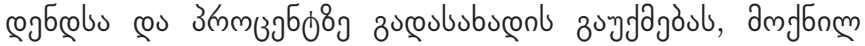

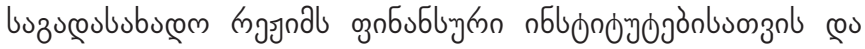

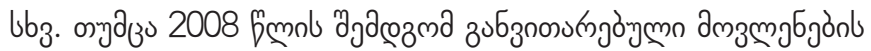

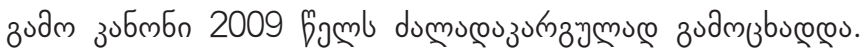

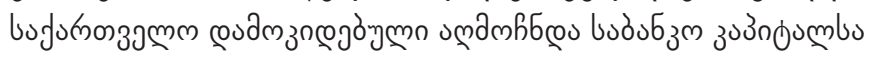

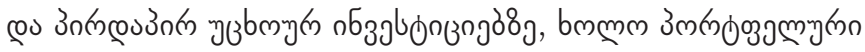

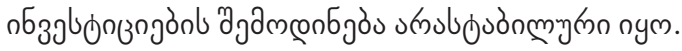

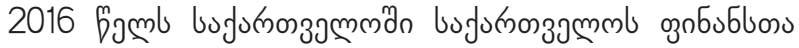

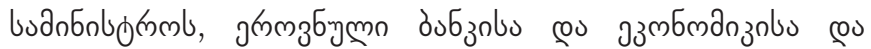

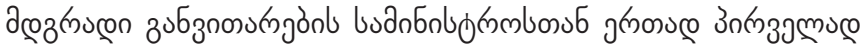

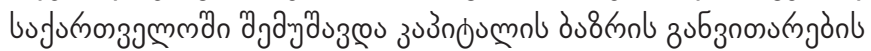

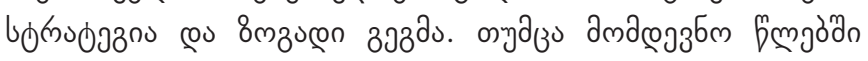

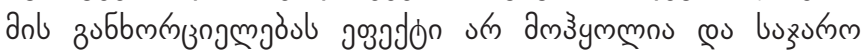

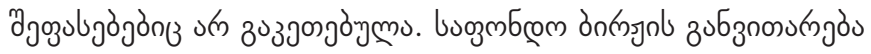

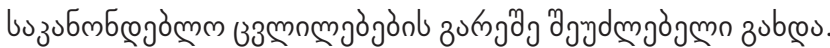

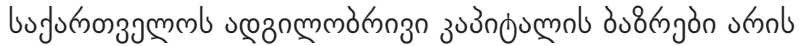

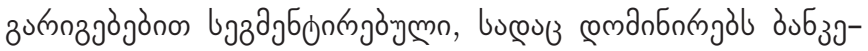

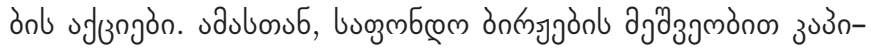

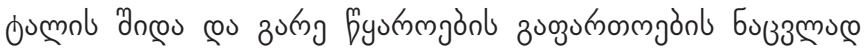

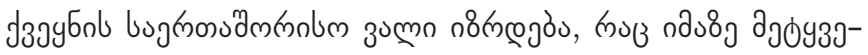

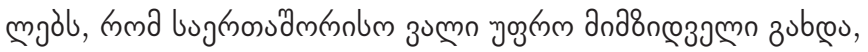

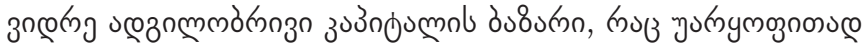

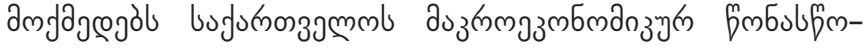

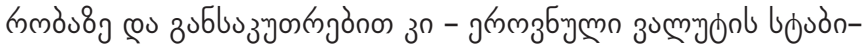

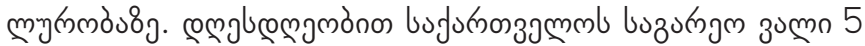

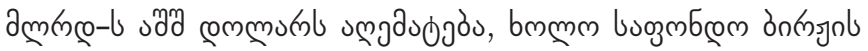

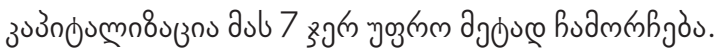

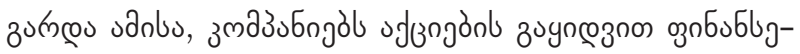

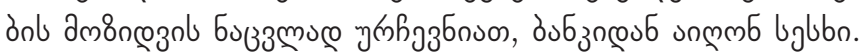

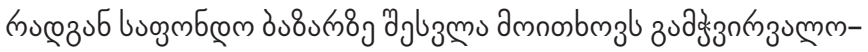

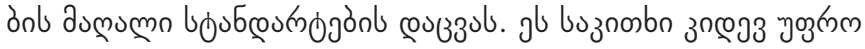

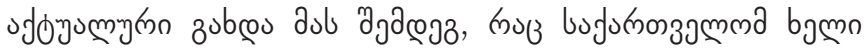
ams

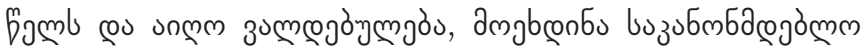

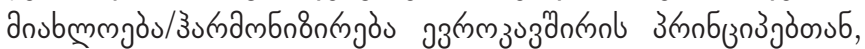

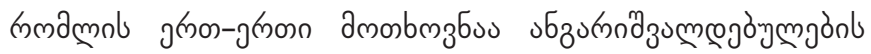

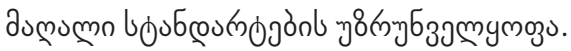

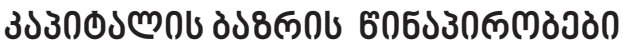

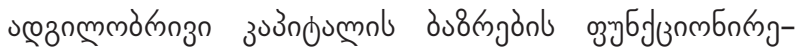

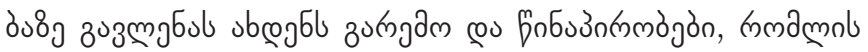

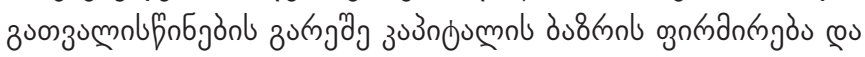

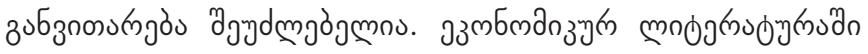

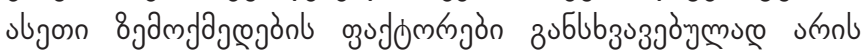

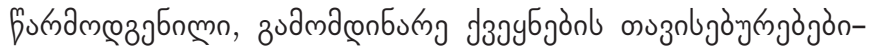

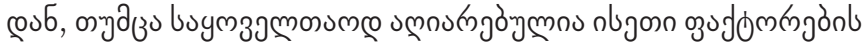

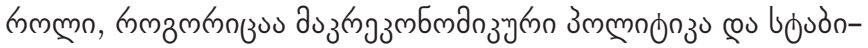

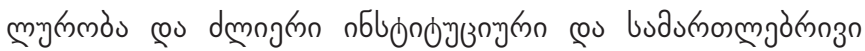




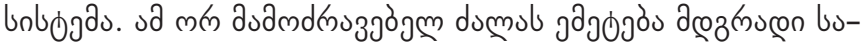

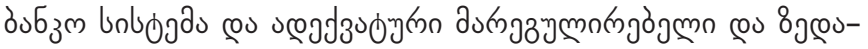

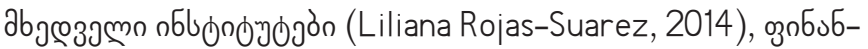

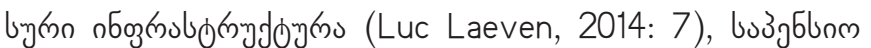

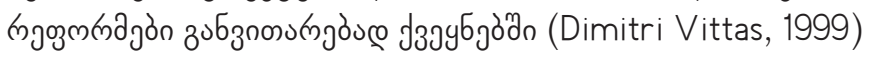
coubro.

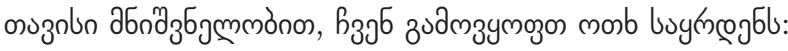

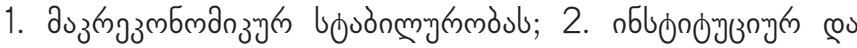

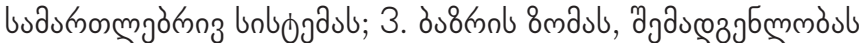

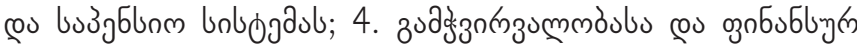

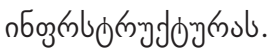

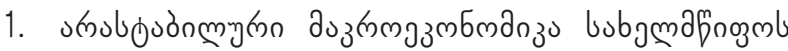

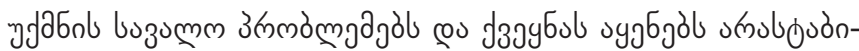

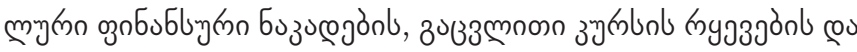

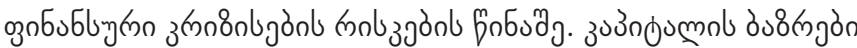

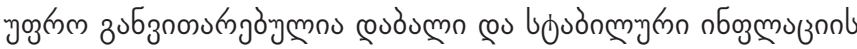

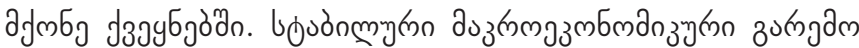

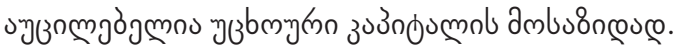

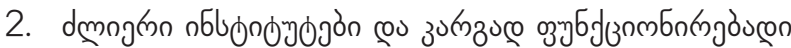

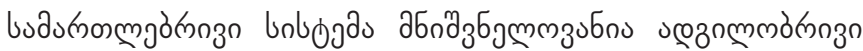

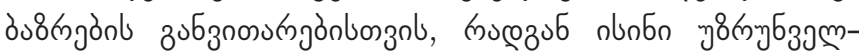

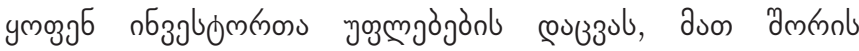

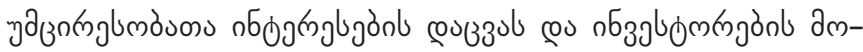

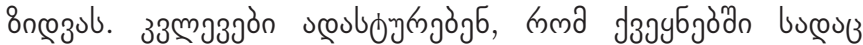

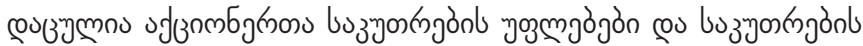

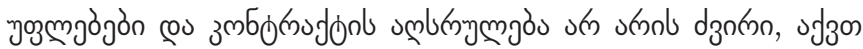

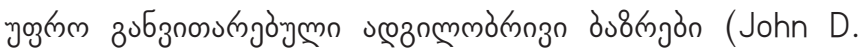
Burger, Francis E. Warnock, Veronica Cacdac Warnock,

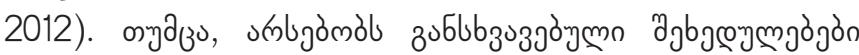

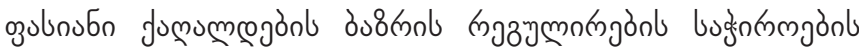

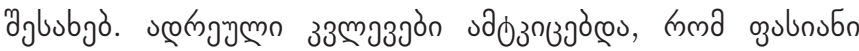

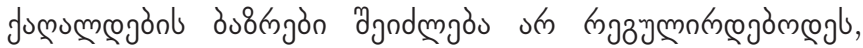

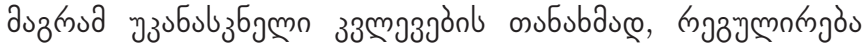

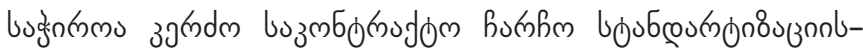

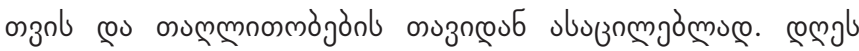

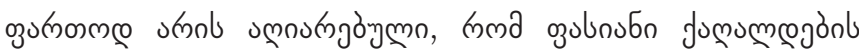

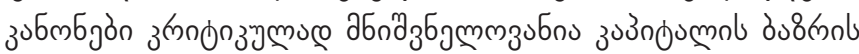

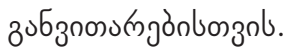

3.

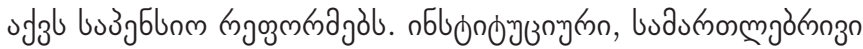
eu nбogmulbf

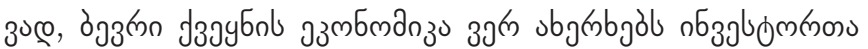
an8n@

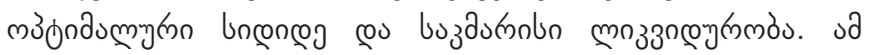

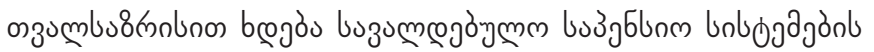

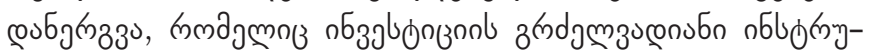

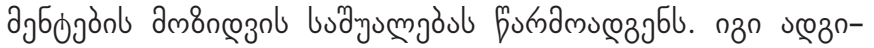

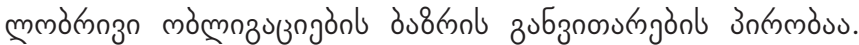

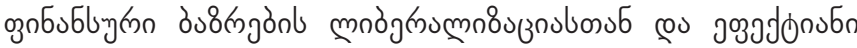

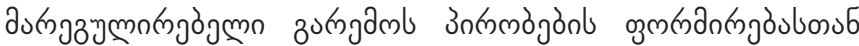

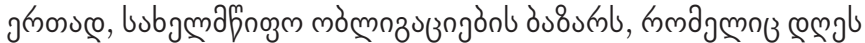

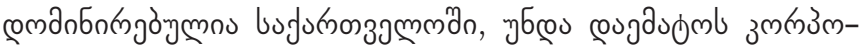

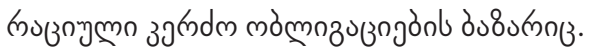

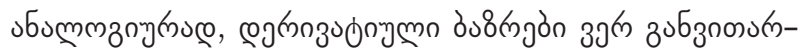

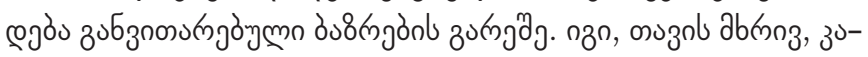
उnou mol bu8

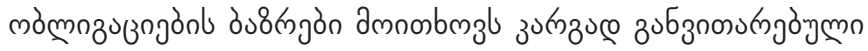

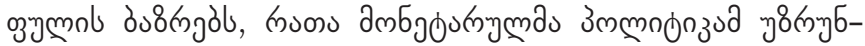

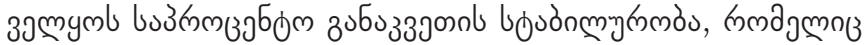

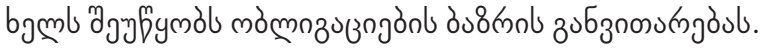

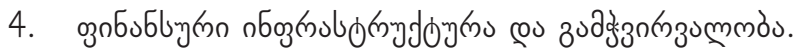

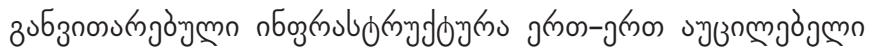

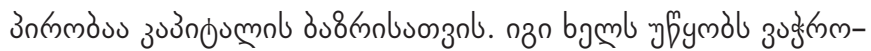

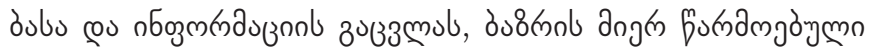

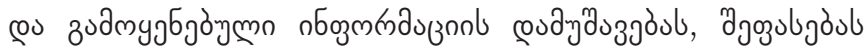

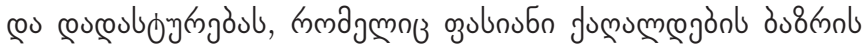

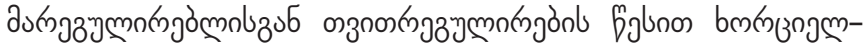

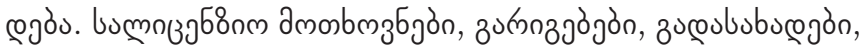

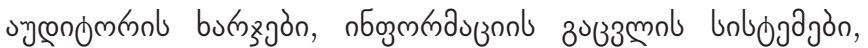

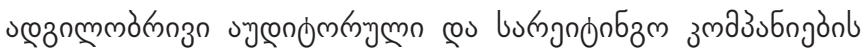

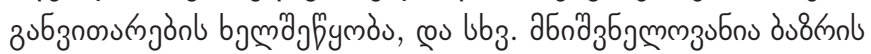

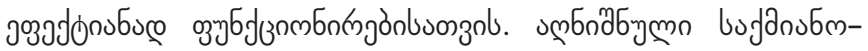

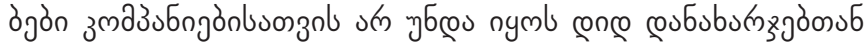

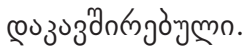

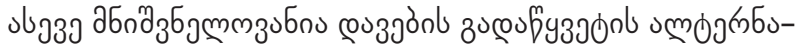

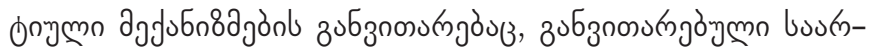

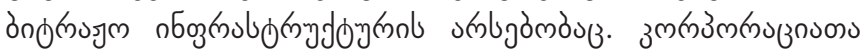

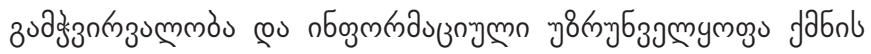

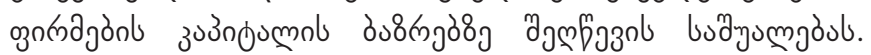

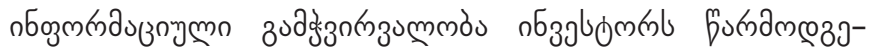

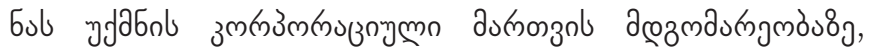

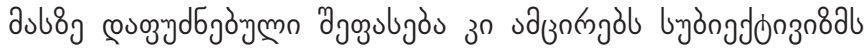

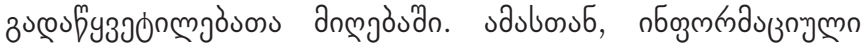
zuałz

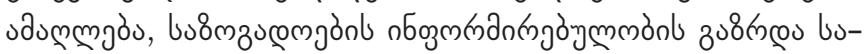

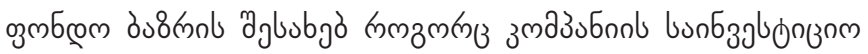
дnд8nœ

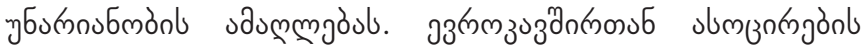

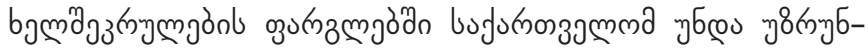

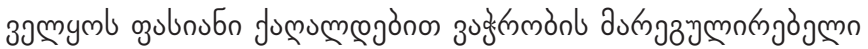

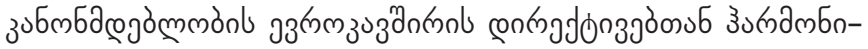

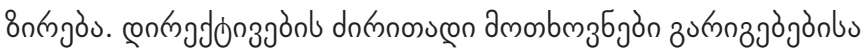

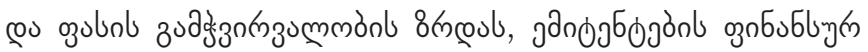

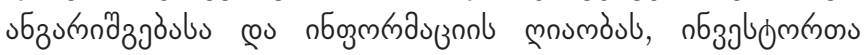

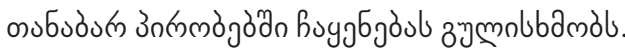

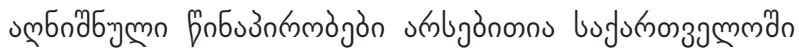

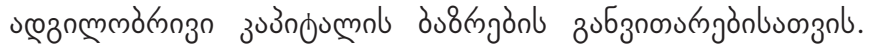

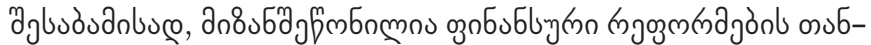

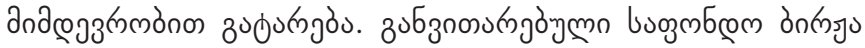

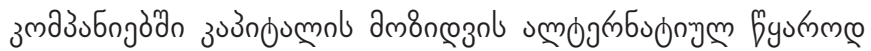




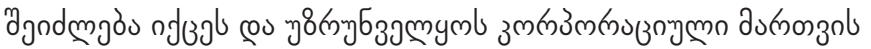

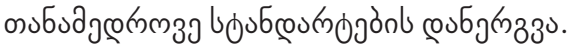

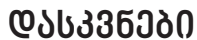

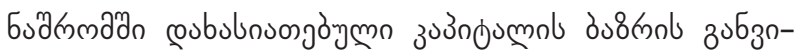

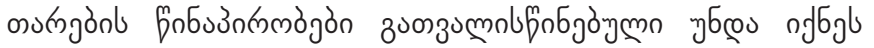

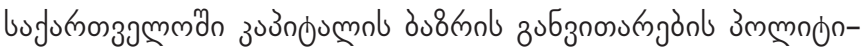
zol zubluogmzmolubl.

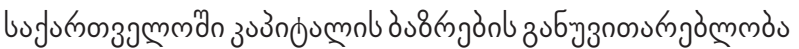

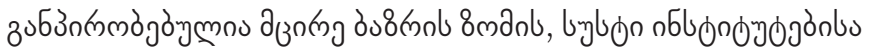

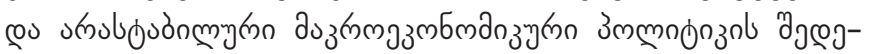

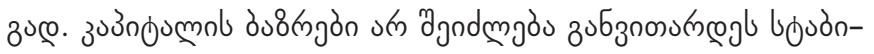

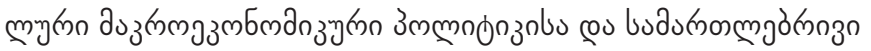

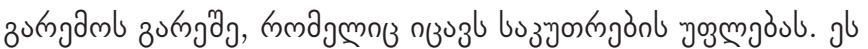

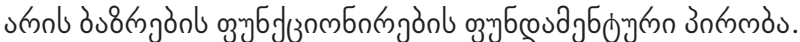

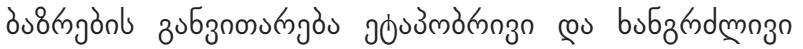

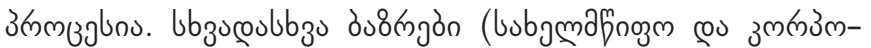

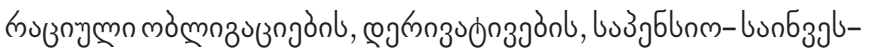

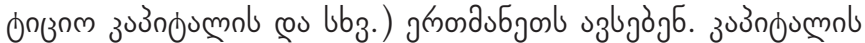

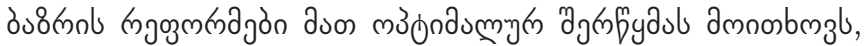

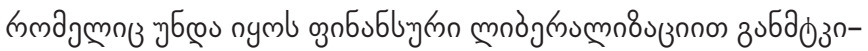

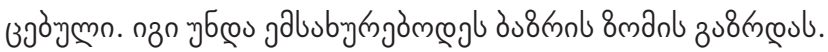

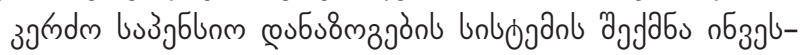

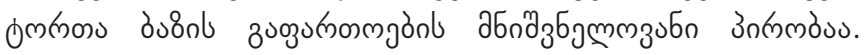

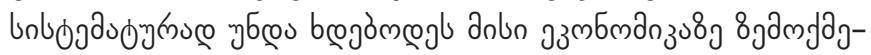

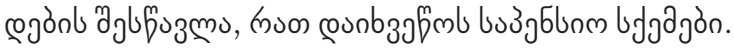

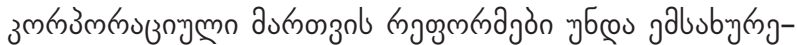
бмल

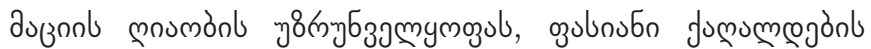

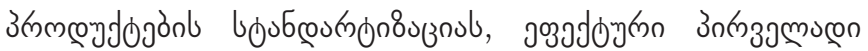

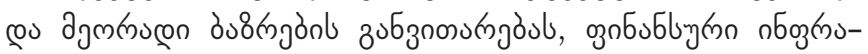

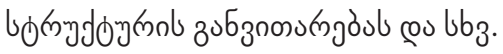

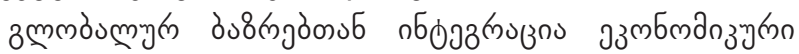

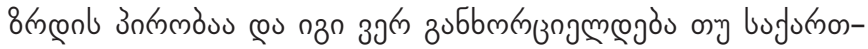

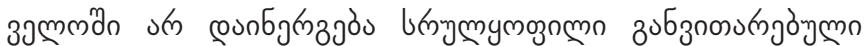

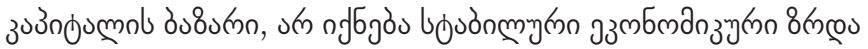

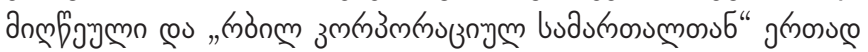

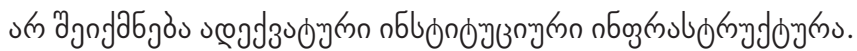

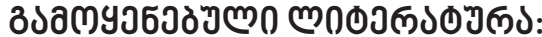

1. Aslanishvili, D. Stock Market in Georgia: Existing Reality and Uncertain Future [Safondo bazari Sakartveloshi: arsebuli realoba da gaurkveveli momavali]. TSU, Science (In Georgian). https://tsu.ge/science/?leng=ge\&lcat=jurnal\&jnomeri=6\&tid=10

2. Ayadi, R., Arbak, E., Naceur, S. B., \& De Groen, W. P. (2013), Financial development, bank efficiency, and economic growth across the Mediterranean. MEDPRO Technical Report, No. 30/March, https://www.files.ethz.ch/isn/161854/ Growth\%20\&\%20Development.pdf;

3. Arcand, J. L., Berkes, E., \& Panizza, U. Too much finance, International Monetary Fund, IMF Working Paper, WP/12/161, 2012. https://www.imf.org/external/pubs/ft/wp/2012/wp12161.pdf

4. Banking and Capital Markets M\&A Outlook, Delloitte, Will it be a stellar year for banking and capital markets mergers and acquisitions? 2019, https://www2.deloitte.com/us/en/pages/financial-services/articles/banking-securities-mergersacquisitions-outlook.html)

5. Demetriades, P. O., \& Rousseau, P. L. (2015), The changing face of financial development. University of Leicester, Working Paper No. 15/20, https://www.le.ac.uk/economics/research/RePEc/lec/leecon/dp15-20.pdf?uol_r=d307e306

6. Deidda, L., \& Fattouh, B. (2002), Non-linearity between finance and growth. Economics Letters, 74(3), https://www. sciencedirect.com/science/article/pii/S0165176501005717

7. Dimitri Vittas, (1999), Pension Reform and Capital Market Development «Feasibility» and «Impact» Preconditions, World Bank Policy Research Working Paper No. 2414, https://papers.ssrn.com/sol3/papers.cfm?abstract_id=632485.

8. Ductor, L., \& Grechyna, D. (2015), Financial development, real sector, and economic growth. International Review of Economics \& Finance, 37, https://pdfs.semanticscholar.org/ffe7/3ea4f441385897aefd3696010a19ee144732.pdf

9. Grassa, R., \& Gazdar, K. (2014), Financial development and economic growth in GCC countries: A comparative study between Islamic and conventional finance. International Journal of Social Economics, 41(6), https://www.emeraldinsight. com/doi/abs/10.1108/IJSE-12-2012-0232

10. Hyoungsoo Zang, Young Chu Kim, (2007), Does Finnaciao Debelopment precede Griwth? Robinson and Lucas might be right. Applied economics letters, V.14, Issue 1, https://doi.org/10.1080/13504850500425469, https://www.tandfonline. com/doi/abs/10.1080/13504850500425469

11. Philip R., and Gian Maria Milesi-Ferretti, (2011), External adjustment and the global crisis, IMF Working Paper, WP/11/197, International Monetary Fund, https://www.imf.org/external/pubs/ft/wp/2011/wp11197.pdf

12. Law, S. H., \& Singh, N. (2014), Does too much finance harm economic growth? Journal of Banking \& Finance, 41 , https://www.sciencedirect.com/science/article/pii/S0378426613004925?via\%3Dihub, jbankfin.2013.12.020 
13. Liliana Rojas-Suarez, (2014), Strengthening Capital Markets in Emerging Economies: Two Key Issues that the G20 Should Not Miss, Center for Global Develooment, February 21, https://www.cgdev.org/blog/strengthening-capital-marketsemerging-economies-two-key-issues-g20-should-not-miss; https://www.bis.org/publ/bppdf/bispap75c.pdf

14. Luc Laeven, (2014), The Development of Local Capital Markets: Rationale and Challenges, IMF Working Paper, International Monetary Fund, WP/14/234, 2014, https://www.imf.org/external/pubs/ft/wp/2014/wp14234.pdf

15. Monetary Policy Implementation at Different Stages of Market Development, IMF Board Paper, 2004, https://www.imf. org/external/np/mfd/2004/eng/102604.htm.

16. Mhadhbi, K. (2014), Financial development and economic growth: A dynamic panel data analysis. International Journal of Econometrics and Financial Management, 2(2), http://pubs.sciepub.com/ijefm/2/2/1/index.html

17. Milesi-Ferretti, Gian Maria, and Cedric Tille, (2011), The great retrenchment: international capital flows during the global financial crisis, Economic Policy, Vol. 26, https://www.researchgate.net/publication/227658749_The_Great_ Retrenchment_International_Capital_Flows_During_the_Global_Financial_Crisis

18. Narayan, P. K., \& Narayan, S. (2013), The short-run relationship between the financial system and economic growth: New evidence from regional panels. International Review of Financial Analysis, 29, http://dx.doi.org/10.1016/j. irfa.2013.03.012, http://isiarticles.com/bundles/Article/pre/pdf/17029.pdf

19. Jagadish Prasad Bist, (2018), Financial development and economic growth: Evidence from a panel of 16 African and nonAfrican low-income countries, Journal Cogent Economics \& Finance, Volume 6, Issue 1, https://www.tandfonline.com/doi/ full/10.1080/23322039.2018.1449780?scroll=top\&needAccess=true, https://doi.org/10.1080/23322039.2018.1449780

20. John D. Burger, Francis E. Warnock, Veronica Cacdac Warnock, (2010), Emerging Local Currency Bond Markets, NBER Working Paper No. 16249, Issued in August Revised in March 2012, NBER Program(s): International Finance and Macroeconomics, https://www.nber.org/papers/w16249

21. Pradhan, R. P., Arvin, M. B., Bahmani, S., Hall, J. H., \& Norman, N. R. (2017), Finance and growth: Evidence from the ARF countries. The Quarterly Review of Economics and Finance, 66, http://isiarticles.com/bundles/Article/pre/pdf/99041. pdf; http://dx.doi.org/10.1016/j.qref.2017.01.011

22. Ross Levin, (1997), Financial Development and Economic Growth: Views and Agenda, Journal of Economic Literature, Vol. XXXY, June, https://www.researchgate.net/publication/4722931_Financial_Development_And_Economic_Growth_ Views_And_Agenda

23. Rousseau, P. L., \& Wachtel, P. (2008), What is happening to the impact of financial deepening on economic growth? January 21, http://pages.stern.nyu.edu/ pwachtel/images/RW_El_Jan2008.pdf

24. Samargandi, N., Fidrmuc, J., \& Ghosh, S. (2015), Is the relationship between financial development and economic growth monotonic? Evidence from a sample of middle-income countries. World Development, 68, https://econpapers.repec. org/article/eeewdevel/v_3a68_3ay_3a2015_3ai_3ac_3ap_3a66-81.htm

25. Stiglitz, Joseph E., (2002), Globalization and Its Discontents, New York: W.W. Norton, https://www.studocu.com/en/ document/ravenshaw-university/macroeconomics/book-solutions/globalization-and-its-discontents/2022797/view

26. William Wright, Panagiotis Asimakopoulos, Eivind Friis Hamre, (2019), Report: the New Financial global capital markets growth index, January, https://newfinancial.org/report-the-new-financial-global-capital-markets-growth-index/

27. World Bank, https://databank.worldbank.org/data/source/global-financial-development/Type/TABLE/preview/on\#advancedDownloadOptions 


\title{
CAPITAL MARKET CHALLENGES AND DEVELOPMENT PREREQUISITES IN GEORGIA
}

\author{
MIKHEIL TOKMAZISHVILI \\ Doctor of Economics, Associate Professor \\ Ivane Javakhishvili Tbilisi State University; \\ Professor, \\ Tbilisi Open University, Georgia \\ M-Tokmazishvili@yahoo.com
}

\section{KEYWORDS: CAPITAL MARKETS, CAPITALIZATION, MARKET STRUCTURE, FINANCIAL MARKET, ECONOMIC GROWTH.}

For citation: Tokmazishvili, M. (2019). Capital Market Challenges and Development Prerequisites in Georgia, Globalization And Business, №8, pp. 60-67. https://doi.org/10.35945/gb.2019.08.006

\section{SUMMARY}

The article describes the challenges of capital markets, concepts of effects of capital markets' development on the economic growth, the current conditions of the capital market in Georgia, restricting and stimulating factors and preconditions necessary for the expansion of the capital market. Through comparative analysis, the problems and trends of development of capitalization are presented.

The formation of capital market is a long process. It requires the formation of financial instruments, consolidated legal or model norms, market infrastructure and institutions. In the developing countries, and moreover, in the PostSoviet countries with least-developed economy and transformational law, the capital market is undeveloped considering the capacity of economy and its potential benefits. The banking sector's ability to finance the economy is restricted, the demand on investment capital is wide, as a result, with the traditional bank financing, establishment and development of the capital market is considered with any alternative.

The paper analyzes the causes that impact on local capital markets functioning and the prerequisites without which the capital market can not be formed and developed in Georgia. The characteristics of impact factors on the capital market through examining of economic literature are presented.

The strong institutions and the well-functioning legal system are important for local market development, as they provide the protection of investors' rights, including the protection of minority interests and attracting investors. The studies show that the country, where the rights of shareholders are protected and the transaction is not expensive, has more developed local markets, however, there is a different view about the necessity for regulating the securities market. The initial studies argued that the securities market may not be regulated, but according to the recent researches, the regulation is essential for private contractual framework standardization and fraud prevention. Today it is widely recognized that the laws of securities are critical to the development of the capital market;

Finally, the article proposes the structure of market prerequisites that are based on several piles: macroeconomic stability, institutional and legal system, market size, market composition and pension system, transparency and financial infrastructure. Despite the absence of institutional, legal and infrastructural barriers, many economies are unable to attract investors in order to ensure the optimum level of capital market and efficient liquidity. In this regard, the compulsory pension systems are introduced, which is an opportunity to attract the long-term instruments of investment. It is the condition of the development of the local bond market. With the liberalization of financial markets and in the effective regulatory environment, investments in the state bonds that dominate in Georgia today will be added by expansion of the corporate private bonds market. Similarly, the derivative markets cannot be developed without a well-developed market and, in turn, they will contribute to the development of the capital market. Moreover, the bond market requires the well-developed money markets in order to encourage the monetary policy to ensure the stability of the percent rate that will support the development of the bond market.

The developed infrastructure is one of the essential conditions for the functionality of the capital market. It promotes trade and information exchange and provides access to predictable products on the background of a less costly system. The transparency and information provision of corporations is also important, which instantly connects investors with money investors. In the framework of the Association Agreement with the European Union, Georgia shall ensure approach to the legislation regulating trade with securities of the EU guidelines. The main requirements of directives are to increase the transparency of transactions and the price, the financial statements of the issuer and the openness of the information, as well as the equal condition for the investors. 